\title{
The Population Level Impacts of Differential Fertility Behavior of Parents of Children with Autism
}

\author{
Kinga Makovi, ${ }^{a}$ Alix Winter, ${ }^{b}$ Ka-Yuet Liu, ${ }^{c}$ Peter Bearman ${ }^{a}$
}

a) Columbia University; b) Harvard University; c) University of California, Los Angeles

\begin{abstract}
Drawing on population level data of exceptional quality (including detailed diagnostic information on the autism status of sibling pairs of over 3 million different mothers), this study confirms that stoppage is the average fertility response to a child born with autism, thereby reducing observed concordance in sibling pairs and leading to potentially biased estimation of genetic contributions to autism etiology. Using a counterfactual framework and applying matching techniques we show, however, that this average effect is composed of very different responses to suspicion of autism depending on birth cohort, the character of the disorder (severe versus less severe), the gender of the child, poverty status, and parental education. This study also sheds light on when parents suspect autism. We find that parents' fertility behavior changes relative to matched controls very early after the birth of a child who will later be diagnosed with autism.
\end{abstract}

Keywords: autism; fertility; matching; causal inference; effect heterogeneity
Citation: Makovi, Kinga, Alix Winter, Ka-Yuet Liu and Peter Bearman. 2015. "The Population Level Impacts of Differential Fertility Behavior of Parents of Children with Autism." Sociological Science 2: 398-419.

Received: January 24, 2015

Accepted: March 13, 2015

Published: August 10, 2015

Editor(s): Jesper Sørensen, Stephen Morgan

DOI: 10.15195/v2.a19

Copyright: (C) 2015 The Author(s). This open-access article has been published under a Creative Commons Attribution License, which allows unrestricted use, distribution and reproduction, in any form, as long as the original author and source have been credited. (C) (i)
W examine the fertility behavior of mothers of children with autism, using a methodological approach that allows us to estimate the effect of suspicion of a first child's autism status on subsequent fertility. In doing so, we provide an improved foundation for autism recurrence rate estimation and reveal patterns of differential ascertainment, illustrating the importance of considering heterogeneity in the autism status of firstborn children as a "treatment," as well as heterogeneity in its effects. Our results reveal when "stoppage" - a decision not to have subsequent children due to the autism status of one's first child-occurs, and demonstrate the importance of integrating demographic decisions into models of heritability.

Prior literature has examined the fertility decisions of parents of children with autism because of their implications for the estimation of an accurate recurrence rate. Autism is widely believed to be one of the most "genetic" of developmental disorders (Abrahams and Geschwind 2008; Lamb, Bailey, and Monaco 2000; Wang et al. 2009). In the absence of known genetic markers, a classic way to ascertain whether a disorder is genetic is to conduct a family resemblance study where concordance of the disorder in twins is compared to concordance in full and half siblings. Only a few such studies have been conducted with respect to autism, yielding strikingly high estimates for recurrence risk. Using population level data, Liu, Zerubavel, and Bearman (2010a) found concordance rates of 47.5 percent in same-sex twins, 9.7 percent in full siblings, and 3.4 percent in half siblings. The fact that autism is at least partly genetically determined has real consequences. High heritability estimates shape the allocation of research priorities as well as lay understanding, potentially contributing to family-planning decisions. 
Family resemblance studies by definition require restricting the population under study to families with more than one child. If parents react to the birth of a child later diagnosed with autism by deciding not to have more children, as a recent population level study indicates is the case (Hoffmann et al. 2014), they are excluded from such studies by design. Equally problematic, we do not know whether the decision to not have more children is structured by relevant autism risk factors. This simple consideration can play havoc with estimates of recurrence risk if parents' fertility responses to the diagnosis of a disorder are shaped by one of the risk factors for that disorder. If, for example, only parents at high risk of having children with a developmental disorder decide to have children after the diagnosis of such a disorder, then the estimated recurrence risk would be inflated compared to the population level recurrence risk. If, in contrast, parents at higher risk of having children with a developmental disorder choose not to have additional children following the diagnosis of a child, population level recurrence rate estimates would be downwardly biased.

Stoppage rules related to autism were first analyzed empirically in the late 1980s by Jones and Szatmari, who concluded that stoppage existed and that it was more pronounced for the most severe cases (Jones and Szatmari 1988). Recently, stoppage has been shown to be present in a population-based study (Hoffmann et al. 2014). We expand on that work by considering the effect of a firstborn with autism on subsequent fertility, by severity in communication and social skills and across demographic subgroups.

Because autism has no biological or genetic markers, it is not diagnosed at birth. Rather, it must be ascertained with the passage of time based on observed social and communication behaviors in order for it to be diagnosed and in order for it to play a causal role in influencing subsequent fertility behavior. Based on prior literature (Basin and Schendel 2007; Durkin et al. 2010; King and Bearman 2011), the ascertainment of autism occurs differently in different social groups, by birth cohort, poverty status, gender of the child, and educational level of the parents. Consequently, if the parents of children with autism tend to exhibit stoppage, we would expect such effects to vary by social group. In this way, autism can be thought of as a heterogeneous treatment with potentially heterogeneous effects, and such heterogeneous effects in the form of stoppage would have implications with regard to which social groups are having fewer children due to the autism status of firstborns (see Elwert and Christakis 2006 for an illustration of the importance of considering heterogeneous treatment effects with regard to health).

To address the above-mentioned complexities, we investigate parents' fertility decisions in relation to firstborn children later diagnosed with autism at the population level. The empirical significance of assessing stoppage as a causal principle is multifold. First, understanding the temporal structure of stoppage provides a better foundation for recurrence rate estimation. Second, our analyses reveal variation in the timing of autism suspicion across different social groups. As variation in timing of suspicion has implications for subsequent fertility, differential suspicion by social groups has implications for the subsequent pattern of autism incidence. 


\section{Roadmap}

Against this background, we first outline why one might expect stoppage to be a heterogeneous treatment with heterogeneous effects. We then present our population level data and analytic strategy, which relies on a counterfactual framework for estimating the causal effect of a first child's autism status on subsequent fertility. Finally, we present our results and explore their implications for autism recurrence rates, the temporal ascertainment of autism, and fertility rates more broadly by socio-demographic subgroups.

To anticipate our main findings, consistent with recent literature, we show that stoppage rules are at play and that they decrease the likelihood of subsequent fertility among mothers whose first child is diagnosed with autism. Examining stoppage by socio-demographic subgroups reveals that parents of children with autism are more likely to exhibit stoppage when their first child's autism is more severe, when their first child was born in more recent years, when their first child is female, and when the child's mother has higher educational attainment at first birth. We observe important differences in the timing of stoppage across social groups. These differences are likely to indicate differences in age of first suspicion of autism by parents.

\section{Background}

Fertility decisions are notoriously difficult to understand even for the people making them, and they are even harder for researchers to apprehend. Think about your own decisions about having children, when, and how many! When should one start a family, how many children should one have, how should these children be spaced over time, should one try for both boys and girls, and when should one stop having children? These decisions are consciously and unconsciously conditioned by a vast number of cognitive and social factors of varying importance (Bachrach and Morgan 2013): shared schemas regarding childbearing, age, health status, number of siblings, childhood experiences, religious beliefs, and environment; the fertility decisions and experiences of close friends, neighbors, and those in similar or adjacent cohorts; the availability of child care services; perceived financial stability, career opportunities, tax incentives, and so forth. It is impossible to collect data on all of the factors guiding fertility decisions, especially since the frameworks within which parents make their family planning decisions are not static over time.

Therefore, instead of modeling fertility behavior with all of its complexity, we aim to assess stoppage-whether parents stop having more children due to the autism status of the first child-as a causal principle. ${ }^{1}$ This entails the assumption that pregnancies taken to term reveal parents' preferences to have more children. Of course pregnancies taken to term are not equivalent to intended fertility, since many couples give birth to children whose conceptions were not planned. Similarly, many couples that want (more) children are unable to conceive. Nonetheless, the revealed preference assumption is less heroic than it seems, both because contraception and abortion carry less stigma than they once did (Morgan 1996), and because assisted reproductive technology is increasingly available (Hammoud et al. 2009; Robertson 
1994). Most importantly, the assumption should not adversely affect our results, because there is no reason to believe that the distributions of fertility problems or contraceptive failure are different in the groups of otherwise similar mothers whose first children were or were not diagnosed with autism. ${ }^{2}$

Why might parents of children with autism exhibit different patterns of fertility behavior than parents of children without autism? A number of mechanisms could link the autism status of one's child to subsequent fertility, including parents' theories of the causes of autism and the anticipated effects of a child's autism on family life (Herbert and Koulouglioti 2010). To examine this possibility, we turn to responses from the Autism Life Histories Survey (ALHS), an online, semi-structured survey whose purpose was to learn about the process of obtaining an autism diagnosis through the stories of parents of children with Autism Spectrum Disorders (ASD). Embedded in these narratives, some parents explicitly addressed future family planning as well as their experiences with autism and its effects on their families. ALHS participants were recruited through websites, blogs, newsletters, and organizations whose functions relate to ASD in various capacities. Recruitment began in September 2010, and the examples presented in this article include responses that were collected through July 2011 (about 1,500 completed surveys). Given that our survey respondents are not a random sample of parents of children with autism, we do not claim to be able to generalize to the full population of families of children with autism with respect to these experiences. Therefore, instead of selecting the most typical stories, we exploit the variation in the data. Our aim is to use parents' voices to shed light on the motivational processes driving family planning among parents of children with autism.

Parents' beliefs about the causes of autism could lead them to be either more or less likely to have additional children after having a child diagnosed with autism. Despite the growing body of research on autism's etiology, no single cause of autism has been identified. It is reasonable to believe that if parents attribute their child's autism to genetic causes, they may react differently relative to parents who believe that their child's autism is attributable to an avoidable exposure, such as vaccination, environmental toxins, or diet during pregnancy. Parents who believe that autism is genetic may be concerned about recurrence rates and, therefore, link this belief to their fertility decisions. Indeed, one mother reported, "When I talked about my concerns with my psychiatrist and our thoughts about another child, he was sort of discouraging because he said that one of his colleagues has two Asperger's kids and how tough it is" (ALHS). In another study, Selkirk et al. (2009) report that out of the 220 parents of children with ASD who provided written comments about factors related to their family planning decisions, 52 (23.6 percent) explicitly mentioned recurrence risk.

Parents who believe that autism is not genetic but rather caused by an avoidable exposure more often report deciding to have more children after an autism diagnosis. As one parent reported, "Felix did have all of his vaccinations, but his last one before the onset of hand flapping was six months prior. I have not vaccinated my other 4 children"3 (ALHS). Therefore, depending on their theories of the cause(s) of autism, parents may be more or less likely to have subsequent children following a previous child's autism diagnosis. As this mother's comment highlights, the timing of autism 
ascertainment has consequences for the theories parents hold about the causes of autism. Although scientifically disproven (Gerber and Offit 2009; Kaye, del Mar Melero-Montes, and Jick 2001; Rosenberg et al. 2013), many parents believe that a vaccine caused their child's autism because they first noticed symptoms shortly after their child was vaccinated, placing ascertainment at around fourteen months. It is, therefore, important to consider the timing of ascertainment when assessing stoppage as a causal principle, as autism can only affect fertility behavior after a child's autism has been suspected.

The effects of having a child with autism on a family may also influence parents' subsequent fertility decisions. Financial pressures associated with providing care for children with autism are common and likely play a significant role in subsequent fertility decisions. As one mother reported in AHLS, "After crying about how much no one at his public school understood him..., he has found a safe and appropriate setting. He says he's going there the rest of his life, but we are drawing money from our pensions to pay tuition."

In sum, there is a relationship between parents' theories of the causes and effects of autism and their motivational accounts relating to fertility behavior. Yet parents of children who all have the same autism diagnosis have varying theories about the causes and effects of autism. This would suggest that if parents of children with autism do tend to exhibit stoppage, the nature and extent of stoppage within this population may vary. Likewise, there is clear qualitative evidence that parents respond to the resource demands of having a child with autism in many different ways. While most responses point towards stoppage, other responses point towards having more children. For instance, a mother noted, "I think one of the most beneficial decisions we made was to have more children, thus giving David younger siblings" (ALHS).

\section{Data}

California's Birth Master Files (BMF) provide a population-based sample of all mothers (more than 6.5 million) who gave birth to their first children in California from 1992 to 2007. Younger siblings were identified based on unique sets of mothers' (encrypted) maiden names and dates of birth. Ambiguous cases in which multiple mothers appeared to have the exact same (encrypted) maiden names and birth dates, and cases in which the sex of the first child was missing, were dropped, resulting in a dataset of 3,162,024 different mothers (for details on the determination of sibling groups within the BMF, see Liu, King, and Bearman 2010b).

The BMF includes both parents' ages, races, and ethnicities, the number of prenatal care visits, whether the birth was paid for by Medi-Cal (California's Medicaid program), the mother's zip code at the time of the birth, and the sex and birth weight of the baby or babies. In these analyses, we rely on singleton first births.

In California, all children with autism are entitled to services coordinated through the Department of Developmental Services (DDS). Therefore, we use records from the DDS to identify all children with autism in California who received services from the DDS from 1992 to 2010. ${ }^{4}$ These data were linked to the BMF using probabilistic matching (for details on the linkage, see King and Bearman 2011) and include each child's date of first evaluation at the DDS as well as five 
items that measure social and three items that measure communication functioning. We use an equally weighted index of these sets of items to construct age-adjusted social and communication severity scores for each child measured on scales ranging from 1 to 100 (for details on the construction and distributions of the developmental functioning scores, see Fountain, Winter, and Bearman 2012). We consider children with scores below 25 at first DDS evaluation to be low functioning.

\section{Methods}

We face the same methodological challenges as any investigation trying to assess the effect of a treatment (in this case, suspecting that one's first child has autism) in a context where a randomized experiment is infeasible. In order to highlight how the autism status of a child differs from other treatments that might affect fertility, we explicitly formulate a counterfactual and then consider confounding factors that might be related to both the autism status of one's child and the fertility decisions of parents (see Harding 2003 for a similar methodological example).

As noted above, it is crucial to consider the timing of parents' ascertainment of autism when assessing the causal impact of a child's autism status on subsequent fertility. Therefore, the proper counterfactual for parents of children with autism is: what would the same parents have decided about subsequent fertility had they not suspected that their child had autism? Similarly, we may ask of parents whose children do not have autism: what would they have done had they suspected that their child had autism prior to acting upon a decision regarding their subsequent fertility? This marks an improvement on prior work as, thus far, stoppage has been talked about without any reference to the timing of fertility decisions relative to the timing of first suspicion or diagnosis. Additionally, prior work has tacitly assumed that, within the same family, the risks of autism and of having a future pregnancy taken to term are stable (Jones and Szatmari 1988). These assumptions do not hold. Older parents are at elevated risk of having children with autism (Durkin et al. 2008), and older parents, on average, are at lower risk of giving birth to additional children. Our analytical strategy addresses these issues by matching mothers on the most salient socioeconomic and demographic predictors of fertility.

To tackle the fundamental problem of causality (Rubin 1974), one should ideally compare mothers who only differ on whether or not their first child was diagnosed with autism but are the same in all other respects related to both fertility and risk factors for having a child with autism, including demographic and socioeconomic characteristics, availability of resources, and desired family size. Given our data, matching on most of the salient socioeconomic predictors of fertility can be perfectly achieved. This unique situation is due to the fact that we have a population level dataset containing more than 3 million California mothers whose fertility histories have been uniquely identified.

We use a two-step matching strategy. First, we exact match on the following variables: mother's and father's races and ethnicities, mother's and father's educational attainment ${ }^{5}$ at the birth of the first child, whether the mother was foreign born, whether the father's name and age were each reported, whether the delivery was covered by Medi-Cal, whether the mother's zip code at first birth was reported, 
and the first child's birth year and sex. In this way, we exploit the missing structure of the data, as missingness in terms of father's name or age may capture relevant aspects of a family's socioeconomic background and family structure. Parental education and socioeconomic status play known and important roles in fertility decisions (Bollen, Glanville, and Stecklov 2001; Skirbekk 2008), shaping both plans and opportunities. Moreover, these descriptors of parents are also associated with the risk of their children receiving an autism diagnosis (Basin and Schendel 2007; Durkin et al. 2010; King and Bearman 2011; Mandell 2009). Given the fact that there is some evidence of male preference in childbearing (Basu and de Jong 2010), and the fact that autism is much more common in boys than in girls, we take into account the sex of the first child as well. The birth year of the first child captures the state of the autism epidemic. Since awareness of autism has changed over time (King and Bearman 2011), it is important to compare mothers in the same environment with respect to the rise of autism. Exact matching establishes a set of possible controls for almost every mother ${ }^{6}$ whose first child was diagnosed with autism.

Final matches were selected from the set of possible control mothers after exact matching (similar to coarsened matching, though in the present case coarsening was not necessary given the number of potential controls; see Iacus, King, and Porro 2012). Controls were selected to approximate the distributions of parental age, birth weight of the first child, number of prenatal care visits, and property values at the zip code level in the case population. ${ }^{7}$ Maternal age is important to consider, because older mothers have fewer opportunities to conceive a second time, and older mothers' children carry a higher risk of being diagnosed with autism. The same holds true for father's age (Croen et al. 2007; Durkin et al. 2008). Low birth weight is associated with higher risk of an autism diagnosis and with other early childhood health outcomes that could alter family planning. The number of prenatal care visits indexes a difficult pregnancy, anxiety due to previous miscarriages, or careful planning. All of these conditions may be associated with behaviors that express themselves in family planning, and some have been linked to the autism status of children (Funderburk et al. 1983; Kinney et al. 2008).

The selection of controls was based on a theoretically calibrated distance function. ${ }^{8}$ The distances between cases' values for each item and those of potential controls were calculated as the squares of the differences between the case and control values normalized by the case's values. In order to leverage the exceptionally large pool of potential controls, we chose the four best controls that minimized the distance function for each case, and assigned each chosen control a weight of 0.25. In this way, we constructed a matched sample of cases and weighted controls; if one aggregates the weights, one derives the exact number of cases. ${ }^{9}$

The full set of balance statistics for our matched sample can be found in Figures 5,6 , and 7 in the appendix. With the strategy described above, we achieve balance on the categorical variables; the differences between the means measured in units of standard deviations of the variables for the cases is less than 2.5 percent for each categorical variable. Most of the variables have the exact same distributions in the two groups achieved by exact matching. In terms of the continuous variables, the differences between the means measured in standard deviation units of the cases 
are approximately 5 percent, a benchmark previously used by others (Caliendo and Kopeining 2008). We exceed this limit for two variables: mother's and father's ages, for which our balances are 6 percent and 7 percent, respectively. For comparison, the differences between the means measured in standard deviation units of the case population of these variables before matching were 42 percent and 46 percent, respectively, so the improvement is substantial.

To minimize censoring, we restrict the population to mothers who gave birth to their first child before 2004, resulting in a dataset of birth histories of 11,841 mothers of firstborn children later diagnosed with autism, and their controls, 45,728 mothers (the average weight is 0.26 , and the maximum weight is 2 ). We compare the proportions of mothers who proceeded to have a subsequent child or children among mothers whose first child was later diagnosed with autism to those whose first child did not receive a diagnosis. As previously indicated, however, autism does not manifest itself at birth and is not ascertained at a particular age across the population. Parents suspect autism well before formal diagnosis in most cases (Giacomo and Fombonne 1998; Saint-Georges et al. 2011; Shattuck et al. 2009), but while they anticipate the diagnosis, we do not know exactly when such suspicions arise. By focusing on the temporal pattern of stoppage, we are able to assess when first suspicion occurs for different social groups.

To achieve this, we compare these proportions by gradually dropping mothers from both groups who proceeded to second conceptions taken to term at one month, two months, three months, and so on, up to four years of age of the first child. ${ }^{10} \mathrm{In}$ other words, while moving along the x-axis of the graphs displaying our results in Figures 1, 2, 3, and 4, mothers proceeding to second conceptions are gradually dropped from both the case and control populations, irrespective of the fertility responses of those to whom they were matched. This strategy may introduce some imbalance in the demographic characteristics of the case and control pools as one moves along the x-axis. ${ }^{11}$ With perfect data on the timing of autism ascertainment, this analysis could consist of a single comparison, but absent this data, our strategy provides a series of estimates of the causal effect of the autism status of one's first child on subsequent fertility based on the timing of suspicion. If the autism status of one's first child is inconsequential for future fertility, we should not see a statistically significant difference between mothers' fertility behavior among those whose first child was diagnosed with autism and those whose first child did not receive a diagnosis in the matched sample.

We also conducted these analyses stratified by communication and social functioning, birth cohort, the gender of the first child, the mother's educational attainment at first birth, the mother's race and ethnicity, and the type of health insurance used to pay for the first child's delivery. Confidence intervals were generated via simulation by calculating the share of mothers with subsequent children assuming a Bernoulli distribution with the parameter (the share of mothers who did proceed to second conceptions taken to term, conditional on not having conceived earlier) estimated from the data. When the 95 percent confidence interval around the probability of having a second child among mothers whose first child was diagnosed with autism does not overlap with the 95 percent confidence interval around the estimate for mothers of first children without autism, we detect a significant effect. 

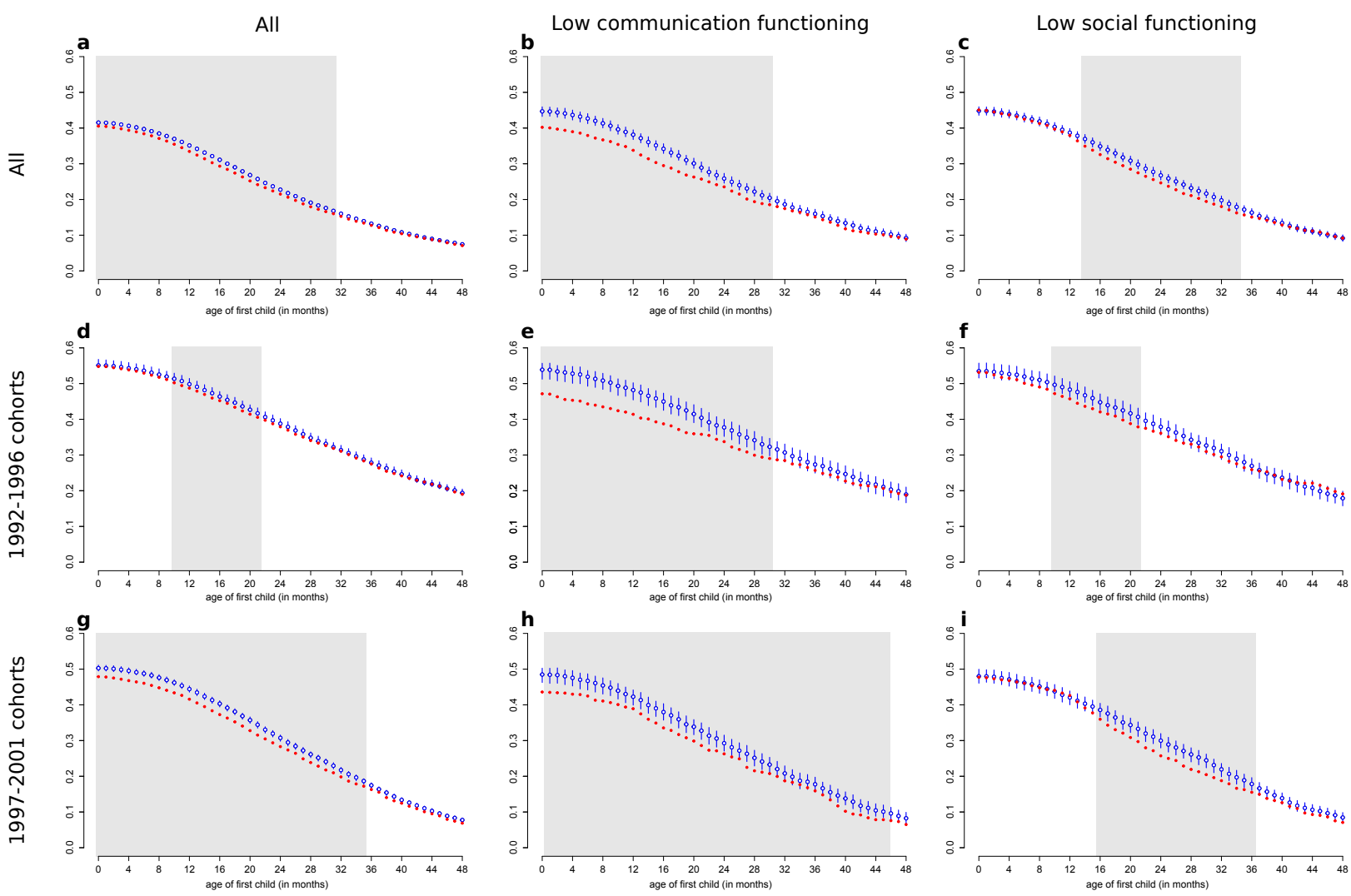

Figure 1: Proportion of mothers of firstborn children with (solid red circles) and without (open blue circles) autism who conceived a subsequent child at each given age of the first child, relative to those who have yet to conceive a second child, stratified by severity group and birth cohort.

Shaded areas indicate significant effects of the first child's autism status on subsequent fertility.

The high quality of our matching eliminates the need for a model-based strategy. However, we used event history models to estimate the effect of ascertainment on future childbearing as a robustness check. ${ }^{12}$ The results of these models are consistent with those described below and are summarized in the appendix.

\section{Results}

We present our results in a set of figures. The x-axes display the age of the firstborn child in months, and the y-axes display the proportion of mothers who conceived a subsequent child or children at each given age of their first child relative to those who have yet to conceive a second child. In other words, the denominator for each proportion includes only the mothers who have not yet conceived another child before the month displayed on the x-axis. The ages at which we ascertain a significant effect are shaded in grey. By definition, as time extends beyond mothers' fertility windows, both these ratios converge to zero. 

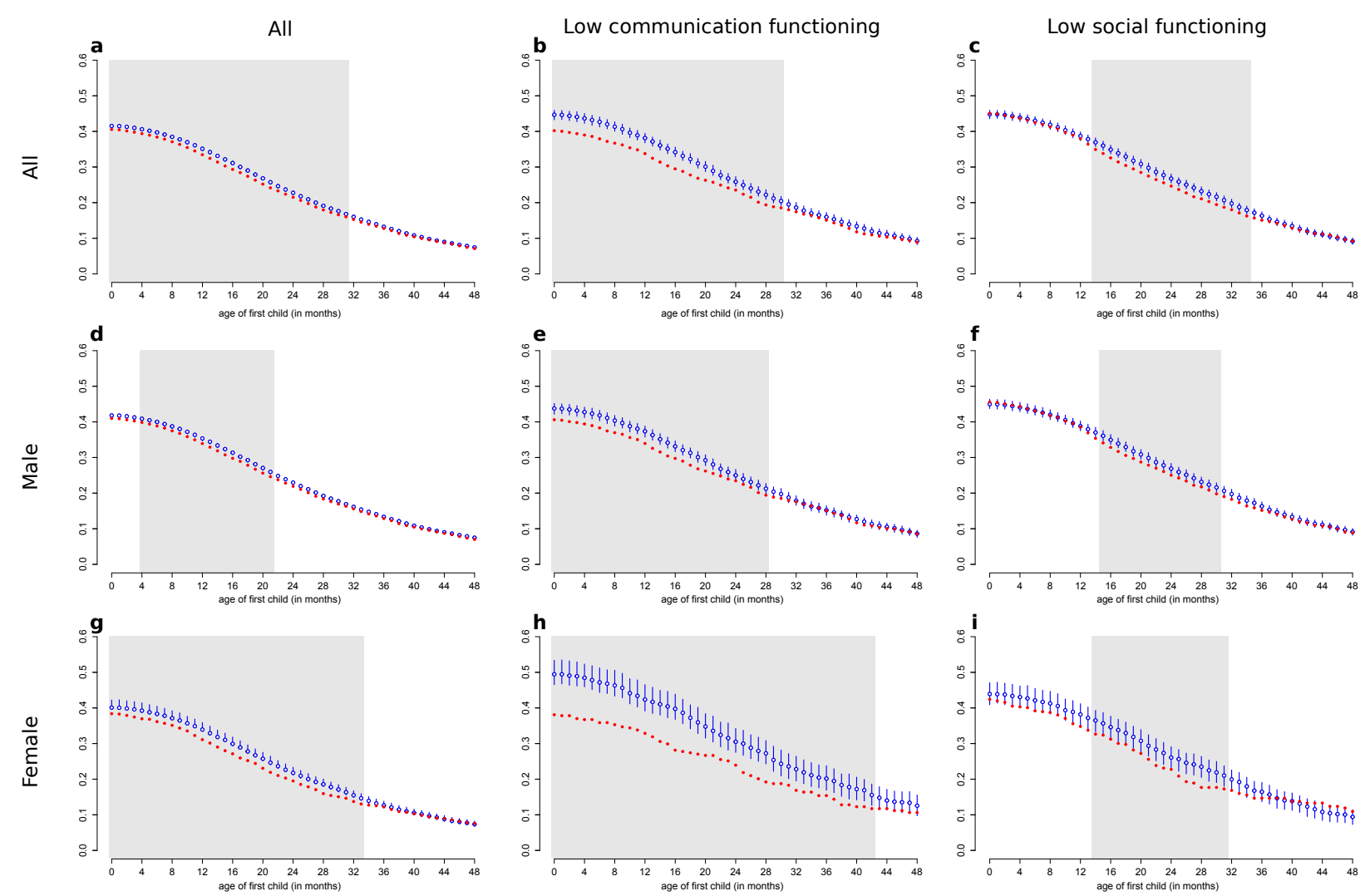

Figure 2: Proportion of mothers of firstborn children with (solid red circles) and without (open blue circles) autism who conceived a subsequent child at each given age of the first child, relative to those who have yet to conceive a second child, stratified by severity group and the gender of the first child.

Shaded areas indicate significant effects of the first child's autism status on subsequent fertility.

Each figure has the same structure. Columns 1-3 report estimates for all children with autism and their controls, children with low communication functioning at first diagnosis and their controls, and children with low social functioning at first diagnosis and their controls, respectively. Row 1 reports estimates for all children, and subsequent rows for social demographic subsets. Blue represents the control population, and red the case population. In Figure 1, we report estimates for early and more recent birth cohorts in rows 2 and 3, respectively. In Figure 2, we report estimates stratified by the first child's gender. In Figure 3, we report estimates by mothers' educational attainment at first birth. In Figure 4, we report estimates by race/ethnicity and Medi-Cal receipt (a crude measure of poverty status).

When comparing mothers whose first children were diagnosed with autism to otherwise similar mothers whose first children were not diagnosed with autism, the order of magnitude of the estimate of autism's effect on subsequent fertility varies with the age of the first child at which first suspicion of autism occurs. Figure 1 shows that, within our full population, autism has a small but significant negative effect on subsequent fertility from the time of the birth of a child later diagnosed 

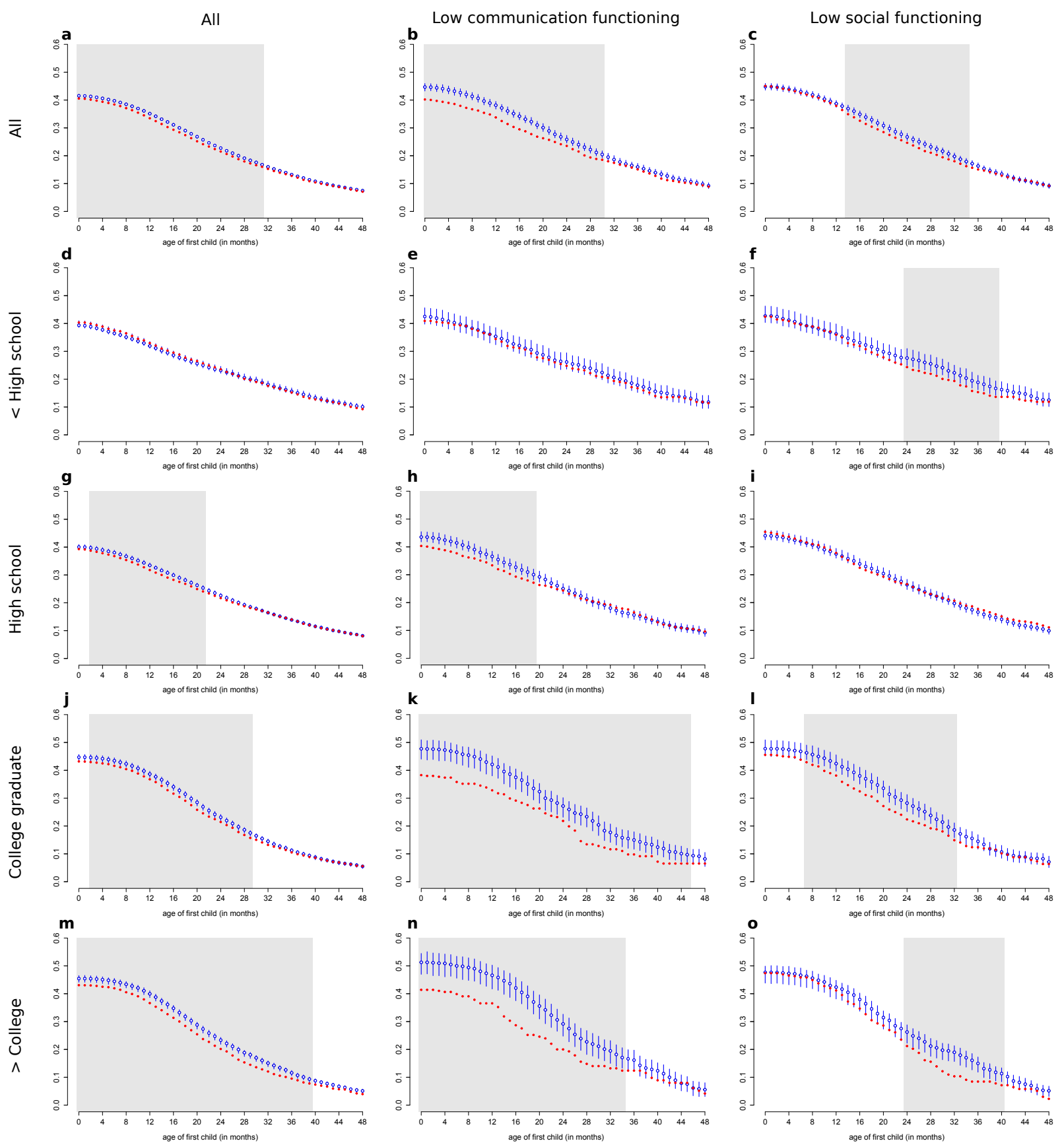

Figure 3: Proportion of mothers of firstborn children with (solid red circles) and without (open blue circles) autism who conceived a subsequent child at each given age of the first child, relative to those who have yet to conceive a second child, stratified by severity group and maternal education at first birth.

Shaded areas indicate significant effects of the first child's autism status on subsequent fertility. 

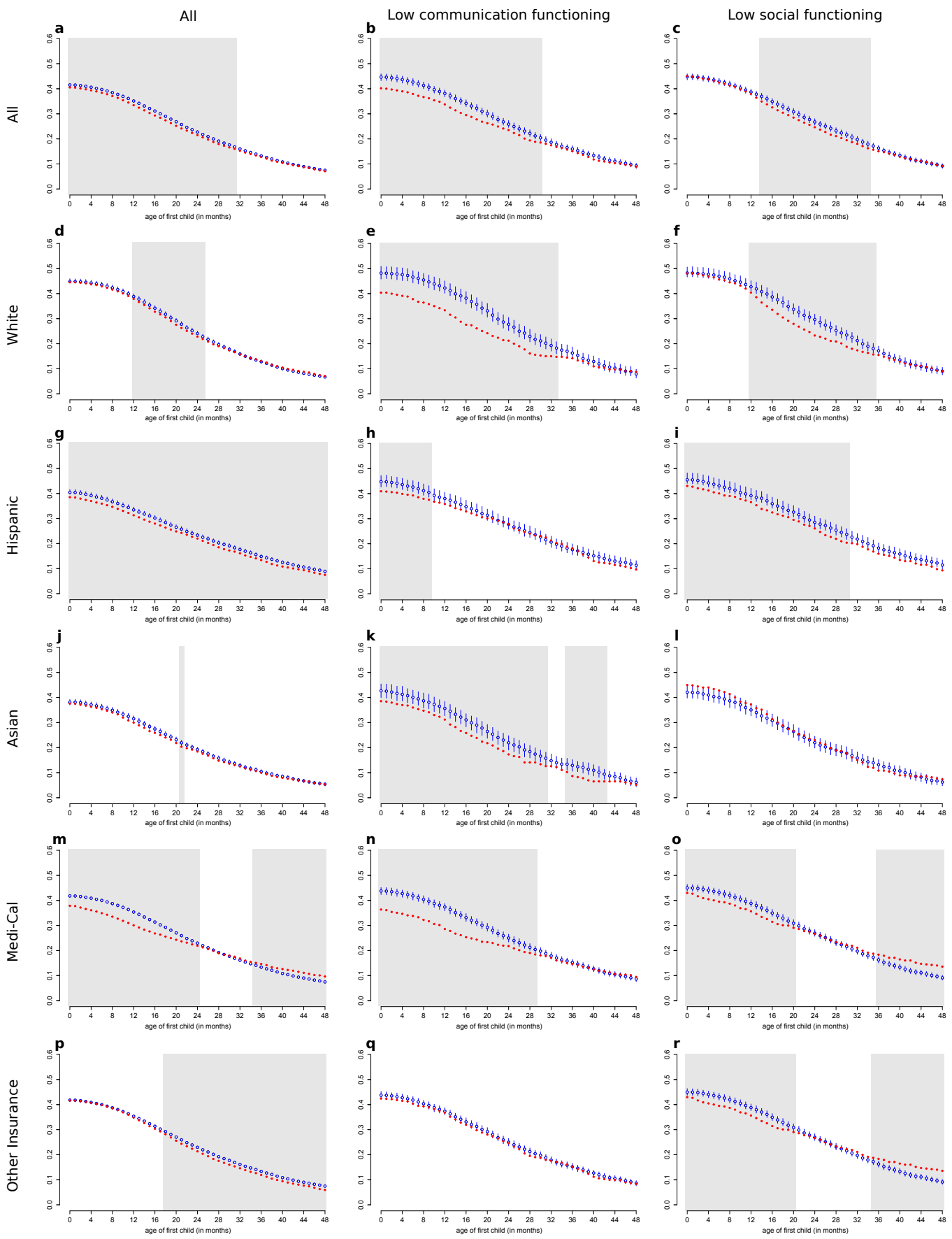

Figure 4: Proportion of mothers of firstborn children with (solid red circles) and without (open blue circles) autism who conceived a subsequent child at each given age of the first child, relative to those who have yet to conceive a second child, stratified by severity group, the race/ethnicity of the mother, and the type of insurance used to pay for delivery.

Shaded areas indicate significant effects of the first child's autism status on subsequent fertility. 
with autism to just under 32 months of age. In other words, mothers of firstborn children later diagnosed with autism are significantly less likely than similar mothers of firstborn children without autism to conceive a second child before the first child reaches 32 months of age. As very few children in our population are diagnosed with autism before the age of three (36 months), the stoppage effect we observe arises from suspicion of a developmental disorder. This finding is consistent with qualitative data that shows that many mothers whose children were subsequently diagnosed with autism felt as if "something was wrong" from a very early age.

Among those whose first child with autism also has low communication functioning, this pattern is substantially more pronounced, especially at younger ages. A similar pattern can be observed among those whose first child with autism has low social functioning, but in this case differences between the behaviors of mothers with firstborns with autism compared to mothers with firstborns not diagnosed with autism are not significant until the first child reaches about 14 months of age. This is consistent with the fact that developmental delays in communication skills are more easily ascertained at younger ages within the immediate family as a child interacts with his or her parents. In contrast, developmental differences in social functioning are more easily ascertained when a child's exposure to the larger community increases, such as through daycare, playgroups, or preschool. These two general patterns-increased stoppage or delay of fertility among parents of children with autism who are lower functioning relative to the full population of children with autism, and stoppage or delay at older ages among those whose children are lower functioning with regard to social behaviors-generally hold across the subsamples described below. This is evident by comparing the figure panels across columns, within rows.

Figure 1 reveals that stoppage or delay is more pronounced in more recent cohorts. As autism awareness has increased with the progression of the epidemic (King and Bearman 2011), parents are more likely to suspect that their child might have autism at younger ages. A negative effect is discernable up to 46 months in more recent cohorts. Among parents of children with low communication functioning, however, stoppage is pronounced in earlier birth cohorts at younger ages. This likely reflects the greater stigma associated with autism in earlier cohorts.

Figure 2 shows that stoppage or delay is more pronounced among parents of firstborn children with autism who are female than among parents of firstborn children with autism who are male, and that stoppage persists among parents of females as their first children age (rows 2 and 3). This is true of the full population, of the subset of parents whose children have low communication functioning, and of the subset of parents whose children have low social functioning. However, the difference between parents of males and parents of females is most pronounced among those whose children have low communication functioning. This is consistent with gendered expectations of child development (Berglund, Eriksson, and Westerlund 2005). A boy with developmentally delayed communication and social skills might be considered "just a boy," while similar behavior exhibited by a girl may be more likely to raise red flags.

Additionally, stoppage or delay is generally more pronounced among mothers with higher levels of education (see Figure 3). In fact, only a small subset of 
mothers with less than a high school degree at first birth whose children have low social functioning exhibit significant stoppage or delay when their first children are approximately 24 to 40 months old. Among the full population and among parents of children with low communication functioning, parents of firstborn children with autism are increasingly less likely to conceive subsequent children when the mother has more years of education at first birth. Among parents of children with low social functioning, autism has no effect on subsequent fertility among mothers with a high school degree but does have an effect among those who are at least college graduates.

Finally, where the sample size is large enough to have confidence in our results, the general pattern we observe overall is upheld when looking at the results by race/ethnicity, with some modest differences (see Figure 4). The qualitative signature of severity, namely, that autism with low communication functioning results in more pronounced stoppage, and that autism with low social functioning exhibits a similar but smaller effect at older ages largely holds, as can be seen from the within-row comparisons. However, Hispanic mothers exhibit a different pattern. This likely arises because the severity distributions for both social and communication functioning for children of white mothers compared to those of Hispanic mothers are significantly different. Namely, children diagnosed with autism who are born to Hispanic mothers are more likely to be lower functioning ( $p$-values of one tailed $t$-tests are $<0.001$, and the differences in means expressed in standard deviations are 10 percent and 33 percent for social and communication functioning, respectively)

Medi-Cal status has previously been used as a measure of socioeconomic background and as a proxy for poverty at the neighborhood level (King and Bearman 2011). Figure 4 shows that mothers whose first birth was paid for by Medi-Cal exhibit more stoppage relative to their respective controls than mothers whose first birth was paid for by private insurance. This is likely due to the different distributions of severity for both communication and social functioning between the two populations ( $p$-values of one tailed $t$-tests are $<0.001$, and the differences in means expressed in standard deviations are 30 percent and 33 percent for social and communication functioning, respectively) as well as the fact that children with autism whose births were paid for by Medi-Cal disproportionately belong to younger cohorts that exhibit more stoppage

\section{Discussion}

On average, the autism status of one's firstborn child has a systematic negative effect on subsequent fertility. This effect is larger among parents of children whose autism is more severe and among certain socio-demographic groups, namely parents of children born in more recent cohorts, parents of firstborn females with autism, and mothers with higher levels of education. These findings are robust despite the fact that our case and control groups may in some instances be quite close to one another. We are unable to distinguish suspicion of autism from suspicion of other developmental concerns. For example, it is possible that parents of some first children not diagnosed with autism observed developmental differences in their children or had 
children with other diagnoses that could have led to stoppage without suspicion of autism. These parents would be in our control group. Therefore, the fact that we observe statistically significantly different fertility patterns for parents of children subsequently diagnosed with autism suggests that our estimates of the effects of suspicion of autism are likely conservative. ${ }^{13}$

Our results provide an improved foundation for estimating the recurrence rate of autism. The subgroup comparisons by mothers' education at first birth indicate that stoppage or delay is more likely among more highly educated women who, given their more advanced age at first birth, have higher risk of giving birth to subsequent children with autism. Consequently, the real recurrence risk may be greater than estimates from concordance studies indicate.

Furthermore, our findings have implications for the timing of autism ascertainment among varying social groups. Our results indicate that the autism status of one's firstborn child behaves as a heterogeneous treatment over time, since autism awareness and autism-related resources have greatly increased since the beginning of the epidemic. One important implication of this observation is that parents of children born in more recent years are more likely to more quickly suspect the autism status of their children and subsequently act with respect to it sooner. Since this is the case, the observed clustering of autism cases within families that was characteristic of the early years of the epidemic will be attenuated. It follows that new autism cases will be distributed among fewer multiplex families. This, in turn, has implications for movements designed to secure resources for children with autism.

The differential results we observe by maternal educational attainment may be either a heterogeneous treatment effect or a heterogeneous effect of the treatment. Namely, more highly educated parents may be more likely to ascertain that their children have autism at younger ages if they are more aware of the disorder, given its historically higher rates of diagnosis among populations of higher socioeconomic status. This would imply a heterogeneous treatment effect of autism. Or, it may be that more highly educated parents are more likely to stop having children in response to the autism status of their firstborn children than parents with lower levels of education, representing a heterogeneous effect of autism. Either way, these differential responses to autism illustrate that autism does not operate uniformly with respect to fertility decisions among all socio-demographic groups. And, either way, these differential responses point to shifts in the population distribution of subsequent autism cases, by socioeconomic status of parents. The attenuation of the clustering of cases within families will not be even across the socioeconomic spectrum, and we can anticipate greater clustering in families with low maternal education. This will have the impact of clustering the autism burden on those least able to absorb it, implicating a need for enhanced state resources.

Similarly parents, unlike community providers (Shattuck et al. 2009), may identify autism more quickly among females than among males, given pervasive gender stereotypes surrounding communication and social interaction, two of the hallmarks of an autism diagnosis. Given these stereotypes, the effect of having a female child with autism appears to be quite different from that of having a male child with autism, resulting in the differential patterns of stoppage we observe. Because 
girls are four times less likely to be diagnosed with autism, the relative rarity of the disorder may also be associated with greater stigma. The pattern of stoppage we observe by gender notably mirrors that for children with severe communication deficits born in the earlier cohort. While this pattern has no implications for the subsequent population distribution of autism cases with respect to gender (the probability of having a male second child is the same following a female first child as a male first child), it suggests that firstborn girls diagnosed with autism will be less likely to have siblings than comparable boys.

Finally, our results have implications for fertility patterns among different sociodemographic subgroups, as we have shown that different subgroups exhibit differential fertility behavior in response to the autism status of a firstborn child. The "unborn children" that are the result of stoppage result in unborn siblings of children with autism, especially in more recent birth cohorts. If autism is in part genetic, differential fertility by socioeconomic status in response to awareness will shift the socioeconomic gradient of autism that we currently observe, aligning autism with other health disorders in which those least advantaged are more likely to be impacted. In this regard, information that leads to stoppage can be seen as a fundamental resource that sustains population level health disparity.

These population level impacts of stoppage have implications for the care of children with autism as they age into adulthood and their parents are eventually no longer able to be their primary guardians. An unanticipated implication of differential stoppage by educational status is that the case population will become more evenly distributed across the socioeconomic spectrum, a process observed already underway in California (King and Bearman 2011). That stoppage is more pronounced for parents on Medi-Cal (an indicator of poverty status) suggests, however, that income and education are playing different roles in driving first suspicion, subsequent ascertainment, and fertility response. While different subgroups generally respond to suspicion of autism in the same way, the picture is clouded by differential ascertainment as indicated by differences in severity status at first diagnosis. Stoppage differences by educational status of parents point to differences in timing of first suspicion; in contrast, severity differences at first diagnosis by poverty status (and the subsequent stoppage effects arising from these differences) point to uneven ascertainment,either arising from fear of state agencies (for Hispanic parents) or uneven access to information on how to navigate the complex DDS service system (for those on Medi-Cal).

A number of potential limitations of our study should be noted. First, our matching of the case and control groups with respect to parental ages is not perfect, but event history models that use mother's age as a control variable show no difference from those models that have no controls (results not shown). Second, our modeling strategy does not make any assumptions with regard to censoring, such as attempting to model moves to outside of California. Consequently, if mothers in the case populations are more prone to moving than controls, we would be less likely to observe second conceptions taken to term in that group, and our results would be biased accordingly. However, since the correlates of moving are similar to the predictors that we match on, there should be no differences between these groups with respect to moving. Furthermore, California provides more extensive autism 
services than its neighboring states and is a leader in service provision nationwide. Therefore, if anything, we would expect mothers whose first child was diagnosed with autism to be less likely to move than otherwise similar mothers, rendering our estimates conservative. Third, mothers should theoretically be removed from the risk set when they make their final decision to not have additional children. Similarly, mothers may also exit and re-enter the risk set at other times when they are temporarily not at risk of a second conception due to a serious illness or the absence of a partner. However, these data are not available.

Net of these potential limitations, this study confirms that stoppage is the average fertility response to a child born with autism (or suspecting that one's child may have a developmental disorder), thereby reducing concordance in sibling pairs and leading, perhaps, to biased estimation of the genetic contributions to autism etiology. This average effect is composed, however, of very different responses to suspicion of autism depending on birth cohort, the severity of the disorder, gender, and parental education. The implication of stoppage following the pattern we observe is that, very slowly, autism will become increasingly characterized by social rather than by communication deficits and will be more evenly distributed across the socioeconomic spectrum.

Equally important, this study sheds light on when parents suspect autism. As recent work has demonstrated, trained clinicians can identify autism in very young infants (Hadley et al. 2010). With respect to their own child, most parents would agree with the idea that their understanding trumps that of others, and so it is not surprising to observe such early evidence of stoppage. We have known for many years that parents routinely suspect autism before others and often feel as if their pediatrician is not responding adequately to their concerns, but we have not had a strategy to capture (at the population level) date of first suspicion. If stoppage arises as a result of suspicion of autism, the simplest message is that parents appear to know that something is different about their child's development very early on. That fact alone has tremendously important implications for our understanding the causes of autism. Consider the simplest case first: before the vaccine theory (Wakefield 1998) was discredited, proponents of vaccines as causing autism would have predicted normal development (and hence no evidence of stoppage) up to roughly 14 months. The evidence presented here clearly refutes this idea. By extension, if autism is ascertained at very early ages, then it follows that exposure to environmental toxicants can only be salient for autism etiology if such exposures pre-date first suspicion. Knowing this makes the search for the environmental determinants of autism significantly more precise, eliminating some candidates and thereby clearing the way for understanding the role of others.

\section{Notes}

1 To be clear, this article is not intended to speak directly to the enormous fertility intentions literature, which would entail a completely different research design. Rather, it is designed to understand the structure of stoppage and the implications of that structure on subsequent understandings of autism etiology. 
2 Readers may wonder if women who give birth using assisted reproductive technologies (ART) are at additional risk of having a child with autism, controlling for those factors (e.g., age, infertility) that lead women to use ART. The brief answer is that most of the additional risk for autism associated with ART arises from multiple embryo transfer (Fountain et al. 2015).

3 Names have been changed to respect the confidentiality of the respondents.

4 As eligibility is determined on the basis of diagnostic parameters without financial or citizenship stipulations, children enrolled with the DDS are very likely to be a random sample of children with autism in California with regard to family planning aspiration prior to diagnosis within social subgroups (Croen et al. 2002).

5 Educational attainment is measured by the highest completed level of education: less than high school; high school graduate (including those with some college education); college graduate; and more than college graduate.

6 There are only 170 mothers whose first child was diagnosed with autism that do not have exact matches for all of the above-mentioned controls. They do not belong to any well defined socio-demographic category.

7 The method used to link property values to our data is described in King and Bearman (2011).

8 The Mahalanobis distance metric was also tried, but it resulted in worse balance for parental age than the distance function described here.

9 We also repeated the analyses described below with three, five, and ten controls. The results do not differ substantially and are therefore not presented here. Additionally, we tried various different weighting structures for the items used at the second matching stage. The theoretically calibrated distance function we ultimately chose-which gives three times the weight to mother's age, and one and a half times the weight to father's age, compared to birth weight and property values, which each have equal weight—resulted in the best balance among many similar alternatives.

10 We calculated the conception dates of mothers' second children by subtracting nine months from the second children's dates of birth.

11 An alternate strategy would be to drop the matched controls when a case-mother proceeds to second conception. However, this would warrant the same strategy when control mothers proceed to second children. This procedure would lead to modifying the sample after the treatment effect is known. It is not justifiable to remove the control mothers matched to a treatment mother after the treatment effect is known, since, by analogy, this would be like removing the control cases of patients for whom the researcher knows the treatment did not work. Rather, the selection of controls has to be done before the trial has begun.

12 The analyses presented in the appendix rely on a sample of 16,874 case-mothers and their 65,194 controls (the average weight is 0.26 , and the maximum weight is 2 ). The difference in the sizes of the two samples arises from our differential treatment of censoring in line with each method.

13 We thank the editors of Sociological Science for this insight.

\section{References}

Abrahams, Brett S. and Daniel H. Geschwind. 2008. "Advances in Autism Genetics: On the Threshold of a New Neurobiology." Nature Reviews Genetics 9(5):341-355. http: //dx.doi.org/10.1038/nrg2346. 
Bachrach, Christine A. and S. Philip Morgan. 2013. "A Cognitive-Social Model of Fertility Intentions." Population and Development Review 39(3):459-485. http://dx. doi .org/10 . $1111 / \mathrm{j} .1728-4457.2013 .00612 . \mathrm{x}$.

Basin, Tanya Karapurkar and Diana Schendel. 2007. "Sociodemographic Risk Factors for Autism in a US Metropolitan Area." Journal of Autism and Developmental Disorders 37(4):667-677. http://dx.doi .org/10.1007/s10803-006-0194-y.

Basu, Deepankar and Robert de Jong. 2010. “Son Targeting Fertility Behavior: Some Consequences and Determinants." Demography 47(2):521-536. http://dx. doi .org/10.1353/ dem.0.0110.

Berglund, Eva, Marten Eriksson, and Monica Westerlund. 2005. “Communicative Skills in Relation to Gender, Birth Order, Childcare and Socioeconomic Status in 18-monthold Children." Scandinavian Journal of Psychology 46(6):485-491. http: //dx. doi . org/10 . $1111 / j .1467-9450.2005 .00480 . x$.

Bollen, Kenneth A., Jennjifer L. Glanville, and Guy Stecklov 2001. “Socioeconomic Status and Class in Studies of Fertility and Health in Developing Countries." Annual Review of Sociology 27:153-185. http://dx. doi .org/10.1146/annurev . soc.27.1.153.

Caliendo, Marco and Sabine Kopeining. 2008. "Some Practical Guidance for the Implementation of Propensity Score Matching." Journal of Economic Surveys 22(1):31-72. http://dx.doi.org/10.1111/j.1467-6419.2007.00527.x.

Croen, Lisa A., Judith K. Grether, Jenny Hoogstrate, and Steve Selvin. 2002. "The Changing Prevalence of Autism in California." Journal of Autism and Developmental Disorders 32(3):207-215. http://dx.doi.org/10.1023/A:1015453830880.

Croen, Lisa A., Daniel V. Najjar, Bruce Fireman, and Judith K. Grether. 2007. "Maternal and Paternal Age and Risk of Autism Spectrum Disorders." Archives of Pediatrics $\mathcal{E}$ Adolescent Medicine 161(4):334-340. http://dx.doi.org/10.1001/archpedi.161.4.334.

Durkin, Maureen S., Matthew J. Maenner, Craig J. Newschaffer, Li-Ching Lee, Christopher M. Cunniff, Julie L. Daniels, Russell S. Kirby, Lewis Leavitt, Lisa Miller, Walter Zahorodny, and Laura A. Schieve. 2008. "Advanced Parental Age and the Risk of Autism Spectrum Disorder." American Journal of Epidemiology 168(11):1268-1276. http: //dx . doi . org/10 . 1093/aje/kwn250.

Durkin, Maureen S., Matthew J. Maenner, F. John Meaney, Susan E. Levy, Carolyn DiGiguseppi, Joyce S. Nicholas, Russell S. Kirby, Jennifer A. Pinto-Martin, and Laura A. Schieve. 2010. "Socioeconomic Inequality in the Prevalence of Autism Spectrum Disorder: Evidence from a U.S. Cross-Sectional Study." PLOS ONE 5:11551. http: //dx.doi.org/10.1371/journal.pone.0011551.

Elwert, Felix and Nicholas A. Christakis. 2006. "Widowhood and Race." American Sociological Review 71(1):16-41. http://dx.doi .org/10.1177/000312240607100102.

Fountain, Christine, Yujia Zhang, Dmitry M. Kissin, Laura A. Schieve, Denise J. Jamieson, Catherine Rice, and Peter Bearman 2015. "Association Between Assisted Reproductive Technology Conception and Autism in California 1997-2007." American Journal of Public Health 105(5):963-971.

Fountain, Christine, Alix S. Winter, and Peter S. Bearman 2012. "Six Developmental Trajectories Characterize Children With Autism." Pediatrics 129(5):e1112-e1120. http: //dx.doi.org/10.1542/peds.2011-1601.

Friedman, Michael. 1982. "Piecewise Exponential Models for Survival Data with Covariates." Annals of Statistics 10(1):101-113. http://dx.doi.org/10.1214/aos/1176345693.

Funderburk, Steve J., Janice Carter, Peter Tanguay, Betty Jo Freeman, and Joan R. Westalke. 1983. "Parental Reproductive Problems and Gestational Hormonal Exposure in Autistic 
and Schizophrenic Children." Journal of Autism and Developmental Disorders 13(3):325-332. http://dx.doi.org/10.1007/BF01531570.

Gerber, Jeffrey S. and Paul A. Offit. 2009. "Vaccines and Autism: A Tale of Shifting Hypotheses." Clinical Infectious Diseases 48(4):456-461. http://dx. doi .org/10.1086/596476.

Giacomo, Andrea De and Eric Fombonne. 1998. "Parental recognition of developmental abnormalities in autism." European Child Adolescent Psychiatry 7:131-136. http://dx. doi . org/10.1007/s007870050058.

Hadley, Darren, Robyn Young, Maria Angelica, Juarez Gallegos, and Carlos Marcin Salazar. 2010. "Cross-Cultural Evaluation of the Autism Detection in Erly Childhood (ADEC) in Mexico." Autism 14(2):93-112. http://dx.doi.org/10.1177/1362361309347676.

Hammoud, Ahmad O., Mark Gibson, Joseph Stanford, George White, Douglas T. Carrell, and Matthew Peterson. 2009. "In vitro fertilization availability and utilization in the United States: a study of demographic, social, and economic factors." Fertility and Sterility 91(5):1630-1635. http://dx.doi.org/10.1016/j . fertnstert. 2007.10.038.

Harding, David J. 2003. “Counterfactual Models of Neighborhood Effects: The Effect of Neighborhood Poverty on Dropping Out and Teenage Pregnancy." American Journal of Sociology 109(3):676-719. http://dx. doi .org/10.1086/379217.

Hebert, Elizabeth B. and Christina Koulouglioti. 2010. "Parental Beliefs about Causes and Course of their Child's Autism and Outcomes of their Beliefs: a Review of the Literature." Issues in Comprehensive Pediatric Nursing 33(3):149-163. http://dx.doi .org/10.3109/ 01460862.2010 .498331$.

Hoffmann, Thomas J., Gayle C. Windham, Meredith Anderson, Lisa A. Croen Judith K. Grether, and Neil Risch 2014. "Evidence of Reproductive Stoppage in Families With Autism Spectrum Disorder-A Large, Population-Based Cohort Study." JAMA Psychiatry. http://dx.doi.org/10.1001/jamapsychiatry.2014.420.

Iacus, Stefano M., Gary King, and Giuseppe Porro. 2012. “Causal Inference Without Balance Checking: Coarsened Exact Matching." Political Analysis 20(1):1-24. http://dx.doi . org/10.1093/pan/mpr013.

Jones, Marshall B. and Peter Szatmari. 1988. "Stoppage Rules and Genetic Studies of Autism." Journal of Autism and Developmental Disorders 18(3):31-40. http://dx . doi .org/10.1007/ BF02211816.

Kaye, James A., Maria del Mar Melero-Montes, and Hershel Jick. 2001. "Mumps, Measles, and Rubella Vaccine and the Incidence of Autism Recorded by General Practitioners: a Time Trend Analysis." BMJ Publishing Group 322(7284):460-463. http://dx . doi . org/10 . 1136/ewjm.174.6.387.

King, Marissa D. and Peter S. Bearman. 2011. "Socioeconomic Status and the Increased Prevalence of Autism in California." American Sociological Review 76(2):320-346. http: //dx.doi.org/10.1177/0003122411399389.

Kinney, Dennis K., Kerim M. Munir, David J. Crowley, and Andrea M. Miller. 2008. "Prenatal Stress and Risk for Autism." Neuroscience E Biobehavioral Reviews 32(8):1519-1532. http: //dx.doi.org/10.1016/j.neubiorev.2008.06.004.

Lamb, Janine A., Janette Moore, Anthony Bailey, and Anthony P. Monaco. 2000. “Autism: Recent Molecular Genetic Advances." Human Molecular Genetics 9(6):861-868. http: //dx.doi.org/10.1093/hmg/9.6.861.

Liu, Kayuet, Noam Zerubavel, and Peter S. Bearman. 2010a. "Social Demographic Change and Autism." Demography 47:327-343. http://dx.doi.org/10.1353/dem.0.0101. 
Liu, Kayuet, Marissa D. King, and Peter S. Bearman. 2010b. "Social Influence and the Autism Epidemic." American Journal of Sociology 115(5):1387-1434. http: //dx. doi .org/ $10.1086 / 651448$.

Mandell, David S., Lisa D. Wiggins, Laura A. Carpenter, Julie Daniels, Carolyn DiGuiseppi, Maureen S. Durkin, Ellen Giarelli, et al. 2009. "Racial/Ethnic Disparities in the Identification of Children With Autism Spectrum Disorders." American Journal of Public Health 99(3):493-498. http://dx.doi.org/10.2105/AJPH. 2007.131243.

Morgan, S. Philip 1996. “Characteristic Features of Modern American Fertility.” Population and Development Review, Supplement: Fertility in the United States: New Patterns, New Theories 22:19-63. http://dx.doi .org/10.2307/2808004.

Robertson, John A. 1994. Children of Choice: Freedom and the New Reproductive Technologies. Princeton: Princeton University Press.

Rosenberg, Rebecca E., J. Kiely Law, Connie Anderson, Carole Samango-Sprouse, and Paul A. Law. 2013. "Survey of Vaccine Beliefs and Practices Among Families Affected by Autism Spectrum Disorders." Clinical Pediatrics 52(9):871-874. http://dx.doi.org/10.1177/ 0009922812438435.

Rubin, Donald B. 1974. "Estimating Causal Effects of Treatments in Randomized and Nonrandomized Studies." Journal of Educational Psychology 66(5):688-701. http://dx. doi . org/10.1037/h0037350.

Saint-Georges, Catherine, Ammar Mahdhaoui, Mohamed Chetouani, Raquel S. Cassel, MarieChristine Laznik, Fabio Apicella, Pietro Muratori, Sandra Maestro, Filippo Muratori, and David Cohen. 2011. “Do Parents Recognize Autistic Deviant Behavior Long before Diagnosis? Taking into Account Interaction Using Computational Methods." PLoS ONE 6:e22393. http://dx.doi.org/10.1371/journal.pone.0022393.

Selkirk, Christina G., Patricia McCarthy Veach., Fengqin Lian, Lisa Schimmenti, and Bonnie S. LeRoy. 2009. "Parents' Perceptions of Autism Spectrum Disorder Etiology and Recurrence Risk and Effects of their Perceptions on Family Planning: Recommendations for Genetic Counselors." Journal of Genetic Counseling 18(5):507-519. http://dx . doi . org/10 . 1007/ s10897-009-9233-0.

Shattuck, Paul T., Maureen Durkin, Matthew Maenner, Craig Newschaffer, David S. Mandell, Lisa Wiggins, Li-Ching Lee, et al. 2009. “Timing of Identification Among Children With an Autism Spectrum Disorder: Findings From a Population-Based Surveillance Study." Journal of the American Academy of Child E Adolescent Psychiatry 48(5):474-483. http://dx.doi.org/10.1097/CHI . 0b013e31819b3848.

Skirbekk, Vegard 2008. "Fertility Trends by Social Status." Demographic Research 8(5):145-180. http://dx.doi.org/10.4054/DemRes.2008.18.5.

Wakefield, Andrew J. 1998. "Autism, inflammatory bowel disease, and MMR vaccine." The Lancet 351:908. http://dx . doi .org/10.1016/S0140-6736(05)70322-6.

Wang, Kai, Haitao Zhang, Deqiong Ma, Maja Bucan, Joseph T. Glessner, Brett S. Abrahams, Daria Salyakina, et al. 2009. "Common Genetic Variants on 5p14.1 Associate with Autism Spectrum Disorders." Nature 459(7246):528-533. http ://dx . doi .org/10 . 1038/ nature07999. 
Acknowledgements: We thank Keely Cheslack-Postava, Alexandra Brewer, Christine Fountain, and Soumya Mazumdar and the members of the Bearman-Minkoff group for helpful comments on previous drafts. This research is supported by the NIH Director's Pioneer Award program, part of the NIH Roadmap for Medical Research, through grant number 1 DP1 OD003635-01.

Kinga Makovi: Department of Sociology, Columbia University. E-mail:

kinga.makovi@gmail.com.

Alix Winter: Department of Sociology, Harvard University. E-mail: alixsw@gmail.com.

Ka-Yuet Liu: Department of Sociology, University of California, Los Angeles. E-mail: ka@soc.ucla.edu.

Peter Bearman: INCITE, Columbia University. E-mail: psb17@columbia.edu. 